\title{
Diarreia causada por Clostridium difficile: \\ Recentes avanços
}

Bárbara de Oliveira Moreira*

Luana Silva Pais ${ }^{* *}$

Livia de Almeida Costa**

\section{RESUMO}

A infecção causada por Clostridium difficile (C. difficile), um dos agentes causadores de diarréia aguda e recorrente, tem como principal fator de risco o uso de antimicrobianos. Recentemente, houve um aumento da incidência e da mortalidade desta afecção. Clinicamente, a mesma pode manifestar-se desde um quadro de diarreia aquosa leve até a forma grave de colite pseudomembranosa. O objetivo deste artigo é apontar as mudanças epidemiológicas da infecção pelo $C$. difficile, além de rever fatores de risco, manifestações clínicas, métodos diagnósticos, tratamento e prevenção desta infecção. O aumento na gravidade da infecção causada pelo $C$. difficile é relacionado a uma nova cepa hipervirulenta, BI/NAPI/Ribotipo 027, que apresenta maior capacidade de produção de toxinas. Essa nova cepa, mais virulenta, ainda não foi detectada no Brasil, porém como já foi identificada em outros países da América, alerta para a preocupante capacidade de disseminação universal. Essa revisão é baseada em artigos publicados nos últimos 10 anos, utilizando como base de dados o PubMed e o Scielo (Scientific Eletronic Library Online), com as palavras-chave: Epidemiologia, diarreia, Clostridium difficile e cepa hipervirulenta.

\section{Palavras-chave: Epidemiologia. Diarreia. Clostridium. Cepa hipervirulenta}

\section{INTRODUÇÃO}

Diarreia nosocomial é uma importante causa de morbimortalidade em pacientes hospitalizados. Clostridium difficile ( $C$. difficile) é o agente mais comum de diarreia infecciosa neste ambiente, sendo o principal fator de risco para sua aquisição o uso de antimicrobianos (ERGEN et al., 2009).

A doença associada a este patógeno varia desde quadros diarreicos leves a eventos fulminantes, com formação de pseudomembranas no cólon e megacólon tóxico (CAMACHO-ORTIZ et al., 2009). A epidemiologia da infecção por $C$. difficile vem apresentando importantes mudanças, especialmente a partir da última década, associadas ao surgimento de uma cepa hipervirulenta, denominada BI/NAPI/ Ribotipo 027 (COIA et al., 2009). Desde 2004, foram descritos grandes surtos de diarreia, com casos de colite grave nos Estados Unidos, Canadá e Reino Unido, atribuídos a essa cepa (LECLAIR et al., 2010), a qual apresenta significante patogenicidade com capacidade de disseminação e resistência a vários antimicrobianos, conferindo um importante potencial epidêmico no âmbito hospitalar e, mesmo, comunitário (ARTEAGA et al.,2009).
O objetivo deste artigo é apontar as mudanças epidemiológicas da infecção pelo $C$. difficile, além de rever fatores de risco, manifestações clínicas, métodos diagnósticos, tratamento e prevenção desta infecção.

\section{ReVisão DE Literatura}

O presente estudo consiste em uma revisão de literatura, com busca em bases de dados como PubMed e Scielo (Scientific Eletronic Library Online). No banco de dados do PubMed foram utilizadas as palavras-chave epidemiologia, diarreia e Clostridium difficile, sendo encontrados 658 artigos dos últimos 10 anos, incluindo artigos nos idiomas inglês, português e espanhol. No banco de dados do Scielo, as palavras-chave foram cepa hipervirulenta e Clostridium difficile, sendo encontrados 56 artigos a partir do ano de 2008. Os artigos foram escolhidos através dos títulos e resumos que apresentavam essas palavras-chave e abordavam o tema, e principalmente os aspectos clínicos da colite grave por $C$. difficile, sendo selecionados aqueles que a diarreia era associada à cepa hipervirulenta. $\mathrm{Na}$ análise, realizou-se síntese reflexiva dos estudos,

*Universidade Federal de Juiz de Fora, Hospital Universitário, Residente de Gastroenterologia - Juiz de Fora, MG. E-mail: barbara.oliveiramoreira@, hotmail.com

**Universidade Federal de Juiz de Fora, Acadêmica da Faculdade de Medicina- Juiz de Fora, MG.

***Hospital Universitário da Universidade Federal de Juiz de Fora, Médica da Empresa Brasileira de Serviços Hospitalares (Ebserh) - Juiz de Fora, MG. 
permitindo aprofundar com detalhes nesta patologia e ainda, ampliar conhecimentos da cepa recentemente descoberta.

\section{Discussão}

\subsection{História}

A colite pseudomembranosa foi descrita primeiramente por Finney em 1893 (GERDING, 2009) como uma diarreia associada ao uso de antibióticos, a qual se acreditava que o principal agente etiológico era o Staphylococcus aureus (S. aureus) (BARTLETT, 2008). Em 1974, foi publicado um trabalho que consistiu em um estudo prospectivo "Clindamycin-associated colitis", que evidenciou 10\% dos casos de colite pseudomembranosa associada ao uso da clindamicina, sem relação com o S. aureus, levantando a possibilidade de outro agente etiológico estar associado a esta condição (TEDESCO et al., 1974). Em 1978, foi identificado C. difficile e sua virulência associada à produção de toxinas como o fator causador da colite associada ao uso da clindamicina (GERDING, 2009).

\subsection{Microbiologia e fatores de risco}

O C. difficile é um bacilo gram-positivo, esporulado, anaeróbio obrigatório, comensal, e que pode compor a microbiota intestinal do homem e de outros animais (ROCHA et al., 1999).

A transmissão deste agente pode ocorrer de pessoa para pessoa através da via fecal-oral. Trata-se de um micro-organismo não invasivo, cuja virulência é causada pela produção de toxinas (SURAWICZ et al., 2013). O uso de antimicrobianos altera a microbiota intestinal habitual, ocasionando depleção de algumas cepas de bactérias comensais como Bacteroides, permitindo a proliferação e infecção pelo $C$. difficile (KELLY, 2012).

Atualmente, os antibióticos que mais frequentemente estão relacionados à diarreia associada ao C. difficile são: fluoroquinolonas, penicilinas de amplo espectro e cefalosporinas. No entanto, virtualmente qualquer antibiótico pode predispor a esta colonização, incluindo o metronidazol e a vancomicina, que são os principais medicamentos utilizados no tratamento dessa condição (LAMONT et al., 2013).

Existe uma nítida correlação do risco de diarreia associada ao $C$. difficile com o número e tempo de uso de antimicrobianos em um paciente. Outros fatores de risco para esta infecção incluem hospitalização ou institucionalização prolongada de pacientes, idade $>65$ anos, uso de medicações que reduzem o pH gástrico (inibidores de bombas de prótons e bloqueadores do receptor de histamina H2), cirurgia prévia do trato gastrointestinal, imunossupressão (malignidade, uso de quimioterápicos, transplante de órgãos, cirrose hepática) e doença inflamatória intestinal, especialmente se a inflamação envolve o cólon (DENEVE et al., 2009). Os fatores de risco para desenvolvimento de colites mais graves associados ao C. difficile, incluem doenças malignas, doença pulmonar obstrutiva crônica, terapia imunossupressora e insuficiência renal (SHETH et al., 2013).

O papel dos inibidores de bomba de prótons na infecção por $C$. difficile ainda é controverso, porém sabe-se que a bactéria na forma esporulada é resistente ao ácido gástrico, enquanto que as formas vegetativas são passíveis de serem destruídas pelo $\mathrm{pH}$ ácido do estômago (CRAVO, 2010).

Todos os pacientes hospitalizados que apresentam quadro de diarreia aguda devem ser submetidos à investigação do $C$. difficile. O mesmo é recomendado para pacientes com doença inflamatória intestinal e em indivíduos imunocomprometidos que apresentam quadro de diarreia aguda, mesmo que a infecção seja adquirida na comunidade. A investigação rotineira de pacientes assintomáticos não é recomendada. (SURAWICZ et al., 2013).

\subsection{Epidemiologia}

Recentemente foi observado um aumento da incidência de casos graves de colite pseudomembranosa nos Estados Unidos, Canadá e Europa, sendo coincidente com o surgimento de uma nova cepa hipervirulenta (LAVALLÉE et al., 2009). Esta cepa apresenta vários métodos de tipagem, por análise com endonuclease de restrição (REA) grupo BI, tipo de campo de pulso 1 Norte Americana (NAP1), por eletroforese em gel de campo de pulso (PFGE) e ribotipos 027 (BI/NAP1/027) (KACHRIMANIDOU et al., 2011).

$\mathrm{O}$ C. diffcicile $\mathrm{BI} / \mathrm{NAPI} /$ ribotipo 027 , em relação às cepas já conhecidas, produz 16 vezes mais toxina A e 23 vezes mais toxina $\mathrm{B}$, e é resistente à moxifloxacino e gatifloxacino (SILVA JUNIOR, 2012). Essa maior patogenicidade pode ser devido à deleção do gene TCD C, que regula negativamente a expressão das toxinas e também pela produção da toxina binária, que potencializa a ação dessas toxinas A e B (MONAGHAN et al., 2008).

Em 2002, a Universidade de Pittsburgh, nos Estados Unidos, relatou aumento contínuo da infecção grave por $C$. difficile, com mais de 300 mil casos graves em 2006 (RUPNIK et al., 2009). Também foram relatados surtos em Quebec, no Canadá, acometendo principalmente pacientes com mais de 65 anos (LECLAIR et al., 2010). O aumento da incidência e 
da gravidade dessa condição também foi observado em Maine, Pensilvânia, Nova Jersey, Georgia, Illinois, Oregon e outros hospitais do Norte da América, atribuídos ao BI/NAP1/027 (O'CONNOR et al., 2009).

$\mathrm{Na}$ Europa, a incidência aumentou em associação com surtos, primeiro no Reino Unido e, em seguida, na Holanda, Bélgica e França. Dois grandes surtos foram relatados no Reino Unido em 2003-2004 e 2004-2005 no mesmo hospital pelo ribotipo 027 (KACHRIMANIDOU et al., 2011). As evidências recentes sugerem um declínio na incidência da infecção associada ao C. diffcicile ribotipo 027 no Reino Unido e na Holanda. No entanto, em 2008-2010 os casos de infecções pela nova cepa foram relatados na Austrália Ocidental, Coreia do Sul, Hong Kong e Costa Rica, de forma alarmante, mostrando o potencial que o microorganismo possui de se disseminar pelos continentes (CLEMENTS et al., 2010).

Estudos já relataram a ocorrência desta cepa hipervirulenta em países mais próximos ao Brasil, estimando-se a possibilidade de sua presença na América Latina. Em um estudo no Hospital Universitário do Chile em 2011 foram descritos dois casos graves, ambos pelo ribotipo 027 (HERNÁNDEZ-ROCHA et al., 2012). Esta cepa, entretanto, ainda não foi isolada no Brasil. Outro estudo, realizado em um hospital terciário do Rio de Janeiro, analisou diarreia associada ao $C$. difficile em pacientes internados e não evidenciou toxina binária nas fezes e nem deleção do gene TCD C. Na análise por proteína $\mathrm{C}$ reativa, diferentes ribotipos foram encontrados como ribotipo 135 e 038, sendo considerado o ribotipo 135 exclusivo do Brasil, não observado em nenhum outro país (BALASSIANO et al., 2010).

\subsection{Manifestações clínicas e diagnóstico}

A virulência do $C$. difficile está relacionada com a produção de toxinas TCD A e TCD B, responsáveis pela maior parte das manifestações clínicas (DUBBERKE et al., 2011). No epitélio intestinal, a toxina $\mathrm{A}$ induz liberação de citocinas como a interleucina 8 , além de perda da função de barreira protetora, com subsequente morte celular por apoptose. Estas alterações são caracterizadas histologicamente, por ulceração epitelial focal e exsudato inflamatório (MONAGHAN et al., 2008).

Aproximadamente $30 \%$ das diarreias hospitalares estão associadas à infecção por $C$. difficile. Cerca de $5-15 \%$ dos adultos saudáveis apresentam anticorpos $\mathrm{IgG}$ contra a toxinas A do $C$. difficile, sendo portadores assintomáticos desse microrganismo. Dentre os pacientes que apresentam sintomatologia, as manifestações clínicas incluem diarreia, dor abdominal, febre, desidratação e distúrbios hidroeletrolíticos, além de distensão abdominal, hemorragia digestiva e até mesmo choque séptico (LAMONT et al., 2013).

A síndrome diarreica pode variar desde diarreia aquosa leve, colite com ou sem a formação de pseudomembranas, até apresentações mais graves com colite fulminante (BUJANDA et al., 2009). Nas formas mais graves, observamos hipoalbuminemia (albumina $<3,0 \mathrm{mg} / \mathrm{dL}$ ) e um dos seguintes achados: leucocitose (leucócitos > 15.000 células $/ \mathrm{mm} 3$ ) ou dor abdominal sem critérios de doença complicada. A diarreia associada ao $C$. difficile quando complicada pode manifestar-se com hipotensão arterial, febre (temperatura axilar $>38,5^{\circ} \mathrm{C}$ ), íleo metabólico com distensão abdominal significativa, alteração do estado mental, leucócitos $>35.000$ células $/ \mathrm{mm} 3$ ou $<2.000$ células $/ \mathrm{mm} 3$, Lactato $>2,2 \mathrm{mmol} / \mathrm{L}$ ou qualquer evidência de falência orgânica (SURAWICZ et al., 2013).

Os fatores de risco independentes como preditores de doença grave são: distensão abdominal, leucocitose e hipoalbuminemia, sendo que essa última pode correlacionar com a gravidade da diarreia (TASLIM, 2009). A colite fulminante é uma das complicações mais temidas e pode ter como complicação perfuração intestinal e megacólon tóxico (LAMONT et al., 2013). Deve ser suspeitada em todos pacientes com distensão abdominal e diarreia, além de toxicidade sistêmica grave (SHETH et al., 2013).

O teste diagnóstico padrão-ouro ainda não se encontra bem estabelecido. A coprocultura isoladamente não é suficiente, além disso, nem todas as cepas de $C$. diffcicile produzem toxinas. O ensaio imunoenzimático (EIA) é o método laboratorial mais utilizado, pela facilidade de execução e objetividade dos resultados, e avalia produção das toxinas A e B nas fezes. Uma revisão sistemática demonstrou que a avaliação concomitante das toxinas A e B apresentam sensibilidade de $75-95 \%$ e especificidade de $83-98 \%$ (SURAWICZ et al., 2013), especialmente se múltiplas análises de toxinas nas fezes são realizadas. A técnica de amplificação de ácido nucleico (Polimerase $C$ reaction) para a identificação de toxinas do $C$. difficile tem alta sensibilidade e especificidade (SILVA JUNIOR, 2012), sendo superior ao EIA como padrão para o diagnóstico de diarreia associada ao $C$. difficile. Não são recomendados testes para controle de cura, pois as toxinas podem permanecer nas fezes por até 30 dias após o fim do tratamento (SURAWICZ et al., 2013).

A avaliação endoscópica através de retossigmoidoscopia ou colonoscopia pode ser utilizada para o diagnóstico nos pacientes com

HU Revista, Juiz de Fora, v. 43, n. 2, p. 155-161, abr./jun. 2017 
suspeita de infecção pelo $C$. difficile quando a avaliação de toxinas por EIA ou PCR não estiverem disponíveis ou renderem resultados inconclusivos. $\mathrm{O}$ aspecto endoscópico pode ser normal em pacientes com diarreia leve ou apresentar aspecto de colite inespecífica em casos moderados. $\mathrm{O}$ achado de pseudomembranas colônicas é patognomônico de infecção por $C$. difficile, porém é encontrado na minoria dos casos. Em casos mais graves, a histologia de biopsias colônicas mostra ulceração focal da mucosa associada à ruptura e infiltrado de células inflamatórias com resíduos necróticos cobrindo área da ulceração, a chamada lesão tipo cume ou lesão tipo vulcão (FELDMAN et al., 2014).

\subsection{Tratamento}

Com a emergência do $C$. diffcicile NAP1 em muitos países, houve alteração da história natural da colite, com evidências de recorrências mais freqüentes e evoluções mais graves (PEREIRA, 2014). O tratamento inicial da colite por $C$. difficile consiste na suspensão imediata do antibiótico causador da diarreia, se possível, seguido de suporte básico e início da terapia antimicrobiana específica (LO VECCHIO et al., 2012). Em casos de alta suspeição dessa condição, o tratamento empírico deve ser considerado independente dos resultados dos testes laboratoriais, pois nesse contexto os testes não têm valor preditivo negativo suficiente para excluir a doença (SURAWICZ et al., 2013).

Os principais agentes antimicrobianos utilizados para o tratamento desta infecção incluem o metronidazol, a vancomicina oral e a fidaxomicina (não disponível no Brasil). Estudos comparativos entre estes antibióticos não identificaram diferenças nos resultados da terapia da colite não grave (COHEN et al., 2010). A vancomicina fica reservada para os casos moderados a graves e refratários, por ter alto potencial em aumentar a prevalência de organismos resistentes, principalmente de Enterococos resistentes à vancomicina (VRE), e pelo fato do metronidazol ter custo mais baixo. O metronidazol deve ser utilizado na dose de 250-500mg, três ou quatro vezes ao dia, por 10-14 dias (FELDMAN et al., 2014).

Nos casos graves, tem sido recomendado o uso de vancomicina, a qual permite reduzir a incidência de complicações (ZAR et al., 2007). A administração deve ser oral $(125 \mathrm{mg}, 4$ vezes ao dia, durante 10 a 14 dias), pois a via parenteral não tem efeito, uma vez que esta droga não é excretada pelo cólon. Doses mais altas de vancomicina $(500 \mathrm{mg}$, a cada 6 horas) podem ser recomendadas em casos complicados. A combinação da vancomicina com metronidazol endovenoso também é uma possibilidade para aumentar níveis de concentração fecal de antimicrobianos, sendo utilizado em pacientes com quadros graves (KELLY et al., 2013).

Atualmente, como terapia adjuvante, pode ser administrada vancomicina intracolônica sob a forma de enema, contendo $500 \mathrm{mg}$ vancomicina e 100 $\mathrm{ml}$ de solução salina a cada 6 horas (KELLY et al., 2013). Essa associação é recomendada em pacientes com a forma complicada da doença e naqueles pacientes que as medicações por via oral não atingem determinados segmentos do cólon, como é o caso de pacientes submetidos à colectomia segmentar prévia ou em pacientes que evoluem com íleo paralítico. (SURAWICZ et al., 2013)

Outras opções terapêuticas incluem Fidaxomicina, $200 \mathrm{mg}$ a cada 12 horas, que tem cobertura de cepas BI/NAPI/Ribotipo 027 (SILVA JUNIOR, 2012). Além deste, novas terapias estão sendo testadas como terapia alternativa ao uso de metronidazol e vancomicina, como a nitazoxanida, rifaximina, ramoplanina e tigeciclina (KACHRIMANIDOU et al., 2011).

Agentes antiperistálticos devem ser evitados nesse grupo de pacientes, pelo risco de precipitar megacólon tóxico. Recomenda-se também a profilaxia para tromboembolismo venoso. (SURAWICZ et al., 2013).

Nas formas graves, além da reposição volêmica e correção de distúrbios eletrolíticos, deve-se garantir a manutenção da reserva proteico-calórica do paciente. Não há razão para se interromper a ingestão de líquidos e nutrientes na maioria dos casos. Desta forma, na ausência de íleo metabólico ou distensão abdominal deve-se manter a alimentação oral. Em casos de aporte calórico insuficiente ou intolerância à dieta por via oral é recomendado dieta enteral (PEREIRA, 2014).

Nos casos fulminantes e refratários, a colectomia precoce diminui significativamente a mortalidade. A escolha é colectomia subtotal com ileostomia terminal (LECLAIR et al., 2010). A sobrevida de pacientes tratados com colectomia segmentar é pior do que aqueles tratados com colectomia subtotal, pois geralmente essa condição acomete todo o cólon. (SURAWICZ et al., 2013)

Uma característica especial desta infecção é a elevada taxa de recorrência, entre 20-30\%. Na primeira recorrência da infecção, recomenda-se utilizar o mesmo regime terapêutico realizado no esquema inicial. Caso a recorrência seja grave, o uso da vancomicina deve ser indicado. Numa segunda recorrência, não é recomendado o uso do metronidazol se o mesmo foi utilizado em esquema anterior. Após a segunda recorrência, a fidaxomicina é proposta como a alternativa com o mesmo grau de recomendação da vancomicina, ambas citadas como 
superiores ao metronidazol. Além disso, deve-se considerar o transplante de microbiota fecal neste cenário (PEREIRA, 2014).

Os agentes antimicrobianos por si só podem reduzir a diversidade da microbiota intestinal (disbiose) e o transplante de microbiota fecal deve ser considerado na infecção por Clostridium recorrente e/ou refratária, baseado na capacidade de reconstituir a diversidade da microbiota em longo prazo. (MOSCOSO et al., 2015).

Esse tratamento pode ser administrado através de enemas, colonoscopias ou infusões lentas por sonda nasoentérica. Para selecionar o doador, são necessários alguns critérios, que incluem não ter feito uso de antibiótico nos últimos seis meses, ser imunocompetente, e não ter antecedente de uso de drogas ilícitas, neoplasias ou doença inflamatória intestinal, e ainda devem ser realizados exames como: sorologia para hepatites A, B e C, vírus da imunodeficiência humana (HIV), parasitológico de fezes, pesquisa de Clostridium difficile nas fezes, além de cultura fecal. Após a escolha do doador, o material fecal é coletado e processado em laboratório, sendo a seguir diluído com soro fisiológico a $0,9 \%$, resultando a solução a ser utilizada (GANC et al.,2015). As perspectivas são que, entre as várias espécies da microbiota fecal, possam ser identificadas as que realmente têm importância para o restabelecimento do equilíbrio, com a possibilidade de preparo de cápsulas, tornando o tratamento de casos recorrentes ou refratários mais fácil (PEREIRA, 2014).

Existem limitadas evidências quanto ao uso de probióticos para reduzir a recorrência do quadro. $\mathrm{O}$ uso de imunoglobulina intravenosa, isoladamente, não tem papel no tratamento da diarreia pelo C. difficile, podendo ser útil no contexto de hipogamaglobulinemia (SURAWICZ et al., 2013).

\subsection{Prevenção}

As medidas de prevenção da diarreia associada ao $C$. difficile incluem a lavagem das mãos, limpeza de superfícies e, principalmente, a adoção de critérios rigorosos para a utilização de antibióticos de amplo espectro (ARTEAGA et al., 2009), além da adoção de precauções entéricas universais para todo paciente que apresente suspeita ou confirmação de infecção por este agente.

Os probióticos são microrganismos capazes de colonizar a mucosa colônica, aumentam a sua resistência contra a colonização de bactérias patogênicas. As espécies mais comuns incluem Lactobacillus, Bifidobacterium e o fungo Saccharomyces boulardii. O seu uso na prevenção ou como coadjuvante no tratamento da colite por $C$. difficile ainda é um assunto controverso na literatura, sendo necessários mais estudos sobre o real benefício (PEREIRA, 2014).

Terapias mais recentes têm focado em limitar as modificações da microbiota intestinal e / ou restaurar a microflora para o seu estado pré-mórbido, reduzindo a colonização do trato intestinal por cepas toxigênicas de $C$. difficile e reforçar a resposta imune contra essas toxinas. A prevenção da colonização intestinal de cepas toxigênicas do $C$. difficile pode ser alcançada através da recuperação da microbiota intestinal por transplante da microflora fecal. Outras modalidades como terapias imunológicas, incluindo anticorpos monoclonais e vacinas contra toxinas do $C$. difficile, podem proteger contra recorrências, mas ainda estão com estudos em andamento (KOCIOLEK et al., 2016).

\section{Conclusão}

A diarreia ocasionada pelo $C$. difficile é uma condição comum em ambientes hospitalares. Atualmente, esses surtos podem, em parte, serem decorrentes de uma cepa hipervirulenta, resistente aos antimicrobianos, o que pode modificar a história natural desta infecção. Nessa revisão, ressaltamos o potencial de disseminação continental dessa cepa, visto que teve início nos Estados Unidos, Canadá e Europa, já sendo identificada em países da América do Sul. No Brasil, entretanto, essa cepa ainda não foi isolada, deixando-se dúvidas quanto aos métodos diagnósticos. É importante conhecer as características moleculares e genéticas das cepas que circulam os hospitais de nosso país. Portanto, com a identificação de cepas hipervirulentas do C. difficile, responsáveis por quadros de colite grave, é primordial o diagnóstico e tratamento precoces, além das adoções de precauções entéricas universais neste contexto, de forma a reduzir a morbimortalidade desta infecção, bem como sua disseminação em ambientes hospitalares. 


\title{
Diarrhea caused by Clostridium difficile: Recent Advances
}

\begin{abstract}
Clostridium difficile (C. difficile) is a causative agent of diarrhea and its main risk factor is the use of antimicrobials. Recently, there was an increase in incidence and mortality. Clinical symptoms can manifest from mild watery diarrhea to severe pseudomembranous colitis. The purpose of this article is to make a literature review of C. difficile -associated diarrhea including its recent epidemiological changes. The increase in the severity of infection caused by Clostridium difficile was related to a new hypervirulent strain, BI/NAPI/ribotype 027, with greater capacity for production of toxins, responsible for clinical manifestations. This new strain, more virulent, has not yet been detected in Brazil, but it was already identified in other countries of America, warns the disturbing ability to universal dissemination. This review is based on articles published in the last 10 years, using as database PubMed and Scielo (Scientific Electronic Library Online), with the keywords: Epidemiology, diarrhea, Clostridium difficile and hypervirulent strain.
\end{abstract}

Keywords: Epidemiology. Diarrhea. Clostridium. Hypervirulent strain.

\section{REFERÊNCIAS}

ARTEAGA, A. et al. Riesgo epidémico de la enfermedad asociada a una nueva cepa de Clostridium difficile. Enfermedades Infecciosas y Microbiología Clínica, v. 27, n. 5, p. 278-284, mai. 2009.

BALASSIANO, I. T. et al. An outbreak case of Clostridium difficile-associated diarrhea among elderly inpatients of an intensive care unit of a tertiary hospital in Rio de Janeiro, Brazil. Diagnostic microbiology and infectious disease, v. 68, n. 4, p. 449-455, set. 2010 .

BARTLETT, J. G. The case for vancomycin as the preferred drug for treatment of Clostridium difficile infection. Clinical Infectious Diseases, v. 46, n. 10, p. 1489-1492, mai. 2008.

BUJANDA, L.; COSME, A. Clostridium difficile associated diarrhea. Gastroenterologia y hepatologia, v. 32, n. 1, p. 48-56, jan. 2009.

CAMACHO-ORTIZ, A.; PONCE-DE-LEÓN, A.; SIFUENTES-OSORNIO, J. Enfermedad asociada a Clostridium difficile en América Latina. Gaceta Médica de México, v. 145, n. 3, p. 223-29, mai. 2009.

CLEMENTS, A. CA et al. Clostridium difficile PCR ribotype 027: assessing the risks of further worldwide spread. The Lancet infectious diseases, v. 10, n. 6, p. 395-404, jun. 2010.

COHEN, S. H. et al. Clinical practice guidelines for Clostridium difficile infection in adults: 2010 update by the society for healthcare epidemiology of America (SHEA) and the infectious diseases society of America (IDSA). Infection Control \& Hospital Epidemiology, v. 31, n. 5, p. 431-455, mai. 2010.

COIA, J. E. What is the role of antimicrobial resistance in the new epidemic of Clostridium difficile?. International journal of antimicrobial agents, v. 33, p. S9-S12, mar. 2009.
CRAVO, M.. Diarreia associada a Clostridium Difficile (DACD) num Hospital Central-Uma reflexão urgente!. Jornal Português de Gastrenterologia, v. 17, n. 3, p. 131-132, mai. 2010.

DENEVE, C. et al. New trends in Clostridium difficile virulence and pathogenesis. International journal of antimicrobial agents, v. 33, p. S24-S28, mar. 2009.

DUBBERKE, E. R. et al. The ecology and pathobiology of Clostridium difficile infections: an interdisciplinary challenge. Zoonoses and public health, v. 58, n. 1, p. 4-20, fev. 2011.

ERGEN, E. K. et al. Nosocomial diarrhea and Clostridium difficile associated diarrhea in a Turkish University Hospital. Médecine et maladies infectieuses, v. 39, n. 6, p. 382-387, jun. 2009.

FELDMAN, M. et al. Tratado Gastrointestinal e Doenças do Fígado. $9^{\circ}$ Edição. Rio de Janeiro: Elsevier, 2014.

GANC, A. J. et al. Transplante de microbiota fecal por enteroscopia alta para o tratamento da diarreia causada por Clostridium difficile. Einstein, v. 13, n. 2, p. 338-9, abr. 2015.

GERDING, D. N. Clostridium difficile 30 years on: what has, or has not, changed and why? International journal of antimicrobial agents, v. 33, p. S2-S8, mar. 2009.

HERNÁNDEZ-ROCHA, C. et al. Epidemic Clostridium difficile ribotype 027 in Chile. Emerging Infectious Diseases, v. 18, n. 8, p. 1370 , ago. 2012.

KACHRIMANIDOU, M.; MALISIOVAS, N.. Clostridium difficile infection: a comprehensive review. Critical reviews in microbiology, v. 37, n. 3, p. 178-187, mai. 2011.

KELLY, C. P. Can we identify patients at high risk of recurrent Clostridium difficile infection?. Clinical Microbiology and Infection, v. 18, n. s6, p. 21-27, nov. 2012. 
KELLY, C. P.; LAMONT, J. T. Clostridium difficile in adults: treatment. UpToDate, online, ago. 2013.

KOCIOLEK, L. K.; GERDING, D. N. Breakthroughs in the treatment and prevention of Clostridium difficile infection. Nature Reviews Gastroenterology and Hepatology, v. 13, n. 3, p. 150, fev. 2016.

LAMONT, J. T. Clostridium difficile in adults: clinical manifestations and diagnosis. UpToDate, online, ago. 2013.

LAMONT, J. T. Clostridium difficile in adults: Epidemiology, microbiology, and pathophysiology. UpToDate, online, ago. 2013.

LAVALLÉE, C. et al. Fatal Clostridium difficile enteritis caused by the BI/NAP1/027 strain: a case series of ileal C. difficile infections. Clinical Microbiology and Infection, v. 15, n. 12, p. 1093-1099, nov. 2009.

LECLAIR, M. A. et al. Clostridium difficile infection in the intensive care unit. Journal of intensive care medicine, v. 25, n. 1, p. 23-30, jan. 2010.

VECCHIO, A. L.; ZACUR, G. M. Clostridium difficile infection: an update on epidemiology, risk factors, and therapeutic options. Current opinion in gastroenterology, v. 28, n. 1, p. 1-9, jan. 2012.

MONAGHAN, T.; BOSWELL, T.; MAHIDA, Y. R. Recent advances in Clostridium difficile-associated disease. Gut: journal of the British Society of Gastroenterology, v. 57, n. 6, p. 850860, jun. 2008.

MOSCOSO, F. et al. Fecal microbiota transplantation in recurrent Clostridium difficile infection. Report of one case. Revista médica de Chile, v. 143, n. 4, p. 531-535, abr. 2015.
O'CONNOR, J. R.; JOHNSON, S.; GERDING, D. N. Clostridium difficile infection caused by the epidemic BI/ NAP1/027 strain. Gastroenterology, v. 136, n. 6, p. 1913-1924, mai. 2009.

PEREIRA, N. G. Infecção pelo Clostridium difficile. Jornal Brasileiro de Medicina, v. 102, p. 27-49, set. 2014.

ROCHA, M. F. G.; SIDRIM, J. J. C.; LIMA, A. Â. M. O Clostridium difficile como agente indutor de diarréia inflamatória Clostridium difficile as an inflammatory diarrhea inducer agent. Revista da Sociedade Brasileira de Medicina Tropical, v. 32, n. 1, p. 4752, jan. 1999.

RUPNIK, M.; WILCOX, M. H.; GERDING, Dale N. Clostridium difficile infection: new developments in epidemiology and pathogenesis. Nature Reviews Microbiology, v. 7, n. 7, p. 526, jul. 2009

SILVA JÚNIOR, M. Recent changes in Clostridium difficile infection. Einstein, v. 10, n. 1, p. 105-109, jan. 2012.

SURAWICZ, C. M. et al. Guidelines for diagnosis, treatment, and prevention of Clostridium difficile infections. The American journal of gastroenterology, v. 108, n. 4, p. 478, fev. 2013.

TASLIM, H. Clostridium difficile infection in the elderly. Acta Medica Indonesiana, v. 41, n. 3, p. 148-151, jul. 2009.

TEDESCO, F. J.; BARTON, Robert W.; ALPERS, David H. Clindamycin-associated colitis: a prospective study. Annals of internal medicine, v. 81, n. 4, p. 429-433, out. 1974.

ZAR, F. A. et al. A comparison of vancomycin and metronidazole for the treatment of Clostridium difficile-associated diarrhea, stratified by disease severity. Clinical Infectious Diseases, v. 45, n. 3, p. 302-307, ago. 2007 